\title{
Combined heart transplantation and replacement of atheromatous proximal arch
}

\author{
Yukiharu Sugimura ${ }^{1}$, Arash Mehdiani ${ }^{2}$, Shintaro Katahira ${ }^{1}$, Christina Loberg $^{1}$, Hug \\ Aubin $^{2}$, Artur Lichtenberg ${ }^{3}$, Udo Boeken ${ }^{4}$, and Payam Akhyari ${ }^{5}$ \\ ${ }^{1}$ Heinrich-Heine-Universitat Dusseldorf \\ ${ }^{2}$ Heinrich-Heine-University Düsseldorf \\ ${ }^{3}$ University Hospital Dusseldorf \\ ${ }^{4}$ Dep. of Cardiovascular Surgery \\ ${ }^{5}$ Medical Faculty, Heinrich-Heine University
}

January 7, 2021

\begin{abstract}
Concomitant surgery on the aortic arch with hypothermic cardiac arrest in the setting of heart transplantation (HTX) is extremely rare. A 67-year-old woman who suffered from ischemic cardiomyopathy was evaluated for HTX. Computed tomography showed diffuse soft and hard atheromatous plaques of the entire aorta. Upon allocation of an appropriate donor organ, the ascending aorta and the proximal aortic arch were replaced using hypothermic cardiac arrest at 28 prior to arrival of the donor heart and without clamping of the diseased recipient aorta. After uncomplicated postoperative course the patient was recovered without neurological complications.
\end{abstract}

\section{Introduction:}

Patients receiving orthotopic heart transplantation (HTX) due to ischemic cardiomyopathy (ICM) certainly present with an increased atherosclerotic profile. Occasionally extensive calcification of the ascending aorta is observed in these patients, in which concomitant replacement of the proximal aortic arch represents a valid option for these patients. Herein, we report a case of combined HTX and proximal arch replacement at our institute.

\section{Case Report:}

\section{Medical history}

A female patient at 67-years of age and past history of ICM remained in chronic heart failure (NYHA III) with severely impaired global left ventricular (LV) function (ejection fraction; EF 20\%). Along with the onsite multidisciplinary conference it was decided to evaluate her for HTX/ LV assist device (LVAD). In the screening examinations, however, relevant stenoses of the superior mesenteric artery (SMA) were found. Thus, percutaneous transluminal angioplasty (PTA) was successfully performed at proximal SMA (Fig.1). Moreover, diffusely scattered atheromatous plaques were also detected in the ascending aorta upon CT scan, representing a prohibitive risk for conventional aortic clamping (Fig.2 and Suppl.1). Meanwhile, five months after SMA PTA and after 502 days on the waitinglist an ABO blood group compatible and size-matched donor heart was offered and accepted for her.

\section{Surgery}


Key steps of the surgical procedure are demonstrated in an online supplemental video (Suppl.1). In brief, after cardiopulmonary bypass $(\mathrm{CPB})$ was conducted via the right axillary artery and right femoral vein. Full sternotomy was performed. At 28 core body temperature hypothermic cardiac arrest (HCA) was achieved. After longitudinal aortotomy the aorta was resected up to the brachiocephalic trunk. A significant change of aortic wall with wall thickening and fragile intimal plaque formation was evident. Antegrade brain perfusion via a selective cannula inserted in left common carotid artery was installed. The brain perfusion via right axillary artery was also maintained with clamping of brachiocephalic trunc. Then, in an end-to-end manner, open anastomosis of a $26 \mathrm{~mm}$ vascular prosthesis (Uni-graft, Braun, Melsungen AG, Germany) to the proximal aortic arch was performed, and lower body perfusion was recommenced after 21 minutes of HCA. While awaiting donor organ arrival, rewarming of the recipient to $34^{\circ} \mathrm{C}$ target temperature was started . After the arrival of the donor organ, HTX was performed using the bicaval technique. Finally, donor aorta was anastomosed with the proximal end of the vascular prosthesis (Fig. 3). Cold and warm ischemic time for the donor heart were 143 and 62 minutes, respectively. The uncompicated weaning from CPB was achieved with proper biventricular function under moderate catecholamine and adequate gas exchange function. Total CPB time was 244 minutes.

\section{Postoperative course}

On $1^{\text {st }}$ postoperative day (POD), the patient had a complex focal epilepsy episode. Brain CT showed no cerebral infarction but a small left parietal subdural hematoma that required no further therapy. After that, postoperative course was unproblematic. The patient was discharged from hospital on $49^{\text {th }}$ POD in a good condition. She subsequently passed four weeks of reconvalescence training and returned home without any need for further medical treatment beyond the regular therapy after HTX. Her neurological status is now unremarkable.

\section{Discussion:}

The objective of this paper is to share our experience with combined HTX and aortic surgery for a specific indication of extended aortic calcification in patients awaiting HTX.

Comparing previously published cases of combined HTX and aortic surgery excluding congenital diseases ${ }^{1-8}$, the backgrund of this patient differs substantially. The aortic pathology of all previous patients was aneurysmal degeneration. However, regarding our case, the patient had no aneurysm but significant calcification representing a contraindication for aortic cross-clamping. Thus, We performed combined HTX and proximal aortic arch replacement to allow for a safe aortic clamping of the de novo implanted vascular graft with consecutive HTX performed in a regulr fashion. Our patient experienced a relatively normal postoperative course. In our opinion, concomitant aortic surgery in the setting of HTX could be performable.

As far as the operative technique, in two previous reports, donor ascending aorta was used instead of the vascular graft for ascending aorta replacement ${ }^{1,7}$. Two patients underwent at first HTX followed by aortic surgery ${ }^{1,4}$, probably due to expected prolonged allograft ischemia. In our report, we performed at first ascending aorta replacement, so that the beginning of recipient operation was timely managed considering the transportation time of the donor organ. CPB was initiated by arterial axillay cannulation and femoral venous cannulation to allow immediate cooling of the patient prior to sternotomy, so that after preparation of the aorta and the right atrium $\mathrm{HCA}$ at $28^{\circ} \mathrm{C}$ and aortic arch surgery with open anastomosis become possible before the arrival of a donor organ. In fact, after completion of arch anastomosis, CPB and rewarming was initiated, allowing yet a timely completion of preparation work to allow an adequate total ischemia time.

\section{Conclusion:}

With increasing numbers of elderly HF patients eligible for HTX but with significant aortic pathology a concomitant aortic surgery strategy without aortic cross-clamping seems to be a feasible approach to enable HTX without highly increased risk of preioperative thromboembolic events.

\section{Ethical approval:}


This manuscript followed the principles of the Declaration of Helsinki and the Declaration of Istanbul. The appropriate informed consent was obtained for the publication of this manuscript.

\section{Data availability statement:}

Data sharing is not applicable to this article as no new data were created or analyzed in this study.

\section{References:}

1. Kocher A, Ehrlich M, Khazen C, et al. Repair of an aortic aneurysm at the time of heart transplantation: report of two cases and review of the literature. Paper presented at: Transplantation proceedings1999.

2. Chu S-H, Chien C, Hsu R-B, et al. Combined heart transplantation and resection of dissecting aneurysm of ascending aorta and aortic arch: a case report. Annals of thoracic and cardiovascular surgery: official journal of the Association of Thoracic and Cardiovascular Surgeons of Asia. 2000;6(1):61-64.

3. Rajagopal K, Rogers JG, Lodge AJ, et al. Two-stage total cardioaortic replacement for end-stage heart and aortic disease in Marfan syndrome: case report and review of the literature. J Heart Lung Transplant. 2009;28(9):958-963.

4. Kırali K, Eren E, Mataracı I, Çevirme D. Combined orthotopic heart transplantation and prophylactic ascending aorta replacement in a Marfan patient. Turkish J Thorac Cardiovasc Surg. 2011;19(3):434-436.

5. Wei J, Sue SH, Lee YT, Chang CY. Combined heart transplantation and total replacement of thoracic aorta in Marfan's syndrome with recurrent aortic dissection: a case report. Transplant Proc.2012;44(4):11741175 .

6. Rudez I, Planinc M, Unic D, et al. Concomitant heart transplantation and replacement of ascending aorta and proximal aortic arch. J Cardiovasc Surg (Torino). 2013;54(5):661-662.

7. de Castro JG, Estefania RH, Delgado C, Del Barrio LG, Rabago G. Combined procedure of heart transplantation and ascending aorta replacement. Asian Cardiovasc Thorac Ann. 2016;24(5):455-457.

8. Hobbs R, Gottret JP, Menon R, et al. Concomitant aortic arch reconstruction using deep hypothermic circulatory arrest during heart transplantation. J Cardiovasc Surg (Torino). 2016;57(4):620-623.

\section{Figure legends:}

Fig. 1. Significant stenosis of the superior mesenteric artery (SMA) and percutaneous transluminal angioplasty (PTA). (A) Preoperative contrast enhanced computed tomography (sagittal view) shows the diffuse calcification of the entire aorta with relevant stenosis of SMA (white arrow). (B) Pre-interventional angiography of SMA showed the downstream branches and the proximal stenosis of SMA (white arrow). (C) Post-interventional angiography of SMA with a stent (black arrow).

Fig. 2. Preoperative chest computed tomography shows the presence of diffuse soft and hard atheromatous plaques at the level of the ascending aorta permitting no safe clamping zone. Plaques of ascendens aorta (A), and the aortic arch (B) (white arrow) are demonstrated in axial view. 3D reconstruction of the aorta shows remarkable irregular wall contour as a further expression of the atheroma burden $(\mathrm{C})$.

Fig. 3. Intraoperative findings from completed orthotopic heart transplantation and proximal aortic arch replaceme

\section{Hosted file}

Fig. 1.pdf available at https://authorea.com/users/355890/articles/502886-combined-hearttransplantation-and-replacement-of-atheromatous-proximal-arch

\section{Hosted file}

Fig 2.pdf available at https://authorea.com/users/355890/articles/502886-combined-hearttransplantation-and-replacement-of-atheromatous-proximal-arch 


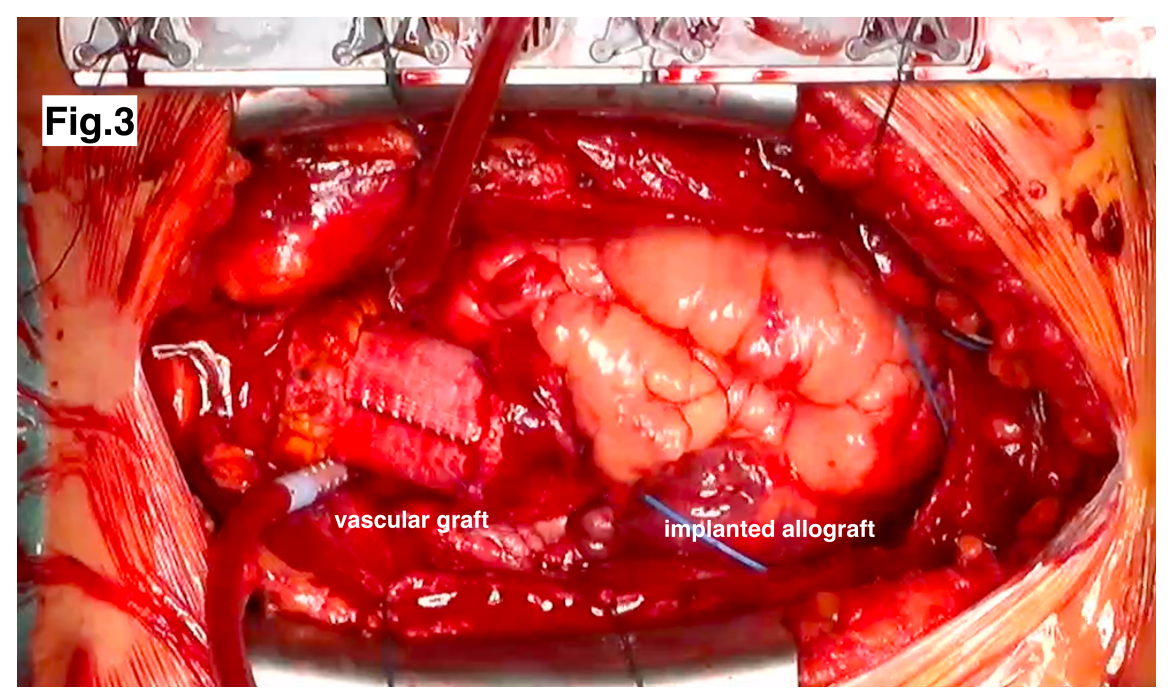

